\title{
A SIMPLE CONSTRUCTION OF MESHES IN APPROXIMATE SYSTEMS
}

\author{
By
}

\author{
N. UGLEŠIĆ
}

\begin{abstract}
Recently, S. Mardešić, L. R. Rubin and T. Watanabe have developed a theory of approximate inverse systems and approximate resolutions, providing thus a new tool to study topological spaces. M.G. Charalambous then introduced a somewhat simpler but more general notion of approximate system. Subsequently, S. Mardešić showed, by a rather general and complicated construction, that the two notions of approximate systems (approximate resolutions) share all relevant properties of their limits (resolutions).

This paper presents a new and rather simple construction with the same properties. Moreover, in the case of topologically complete approximate resolutions, uniqueness up to isomorphisms is established. At the end, it is indicated how one can extend this construction onto approximate mappings.
\end{abstract}

\section{Introduction}

S. Mardešić, L. R. Rubin and T. Watanabe ([5], [7]) have developed a theory of (gauged) approximate resolutions of spaces and mappings, which allows successful study of topologically complete spaces using techniques of (gauged and noncommutative) inverse systems and resolutions. A gauged approximate (inverse) system contains a prescribed collection of normal coverings, called meshes, which controlls the noncommutativity of the bonding mappings and it refines the relevant normal coverings.

Recently M.G. Charalambous ([1]) showed that one can study limits of approximate systems of uniform spaces without meshes, Subsequently, S. Mardešić ([4]) proved that, generally, gauging is not essential for objects, i.e., for approximate systems (resolutions) of Tychonoff (topologically complete)

Key words: Inverse system, approximate (gauged) inverse system, resolution, approximate (gauged) resolution.

AMS (MOS) Subj. Class.: 54B35, 54F45.

Received July 6, 1993. 
spaces. To a given nongauged approximate system $\boldsymbol{X}$, he constructed an induced gauged approximate system $\mathscr{X}$, showing that $\boldsymbol{X}$ and $\mathscr{X}$ share all the relevant limit and resolution properties.

This paper brings a new, more natural and much simpler construction of an induced gauged approximate system. Moreover, in the case of topologically complete approximate resolutions the uniqueness (up to isomorphism) of the induced object is proved.

For the sake of completeness, let us briefly recall the main definitions from [7].

(1.1) Definition. A (gauged) approximate (inverse) system is a collection $\mathscr{X}=\left(X_{a}, \mathcal{U}_{a}, p_{a a^{\prime}}, A\right)$ consisting of :

-a preordered set $A=(A,<)$, which is directed and unbounded;

-for each $a \in A$, a (topological) space $X_{a}$ and a normal covering (mesh) $U_{a}$ of $X_{a}$;

-for each related pair $a<a^{\prime}$ in $A$, a (continuous) mapping $p_{a a^{\prime}}: X_{a^{\prime}} \rightarrow X_{a}$ ( $p_{a a}=1_{x_{a}}$ is the identity mapping on $X_{a}$ ).

The data are to satisfy the following three conditions:

(A1) $\quad\left(p_{a a^{\prime}} p_{a^{\prime} a^{\prime \prime}}, p_{a a^{\prime \prime}}\right)<\mathcal{U}_{a} \quad$ whenever $a<a^{\prime}<a^{\prime \prime}$;

(A2) $\quad(\forall a \in A) \quad\left(\forall q \in \operatorname{Cov}\left(X_{a}\right)\right) \quad\left(\exists a^{\prime}>a\right) \quad\left(\forall a_{2}>a_{1}>a^{\prime}\right)$

$\left(p_{a a_{1}} p_{a_{1} a_{2}}, p_{a a_{2}}\right)<\mathcal{U}$;

(A3) $\quad(\forall a \in A) \quad\left(\forall \mathscr{C} \in \operatorname{Cov}\left(X_{a}\right)\right)\left(\exists a^{\prime}>a\right)\left(\forall a^{\prime \prime}>a^{\prime}\right) \quad U_{a^{\prime \prime}}<p_{a a^{\prime \prime}}^{-1} \mathscr{U}$.

Here, for any two mappings $f, g: X \rightarrow Y$ and any covering $\mathcal{C}$ of $Y,(f, g)$ $\langle\mathcal{V}$ means that, for every $x \in X$, there exists a $V \in \mathcal{V}$ such that $f(x)$ and $g(x)$ belong to $V$. Instead of $(f, g)<\mathcal{V}$ we shall of ten write $f={ }_{c \nu} g$. For coverings $U, U^{\prime}$ of $X, V^{\prime}<U$ means that $U^{\prime}$ refines $U . \quad \mathcal{C o v}_{o v}(X)$ is the set of all normal coverings of a space $X$. If $X^{\prime} \cong X$ and $U \in \mathcal{C}_{o v}(X)$, then the star of $X^{\prime}$ with respect to $U$ is the set

$$
s t\left(X^{\prime}, \mathcal{U}\right)=\cup\left\{U \in \mathcal{U} \mid X^{\prime} \cap U \neq \varnothing\right\} \subseteq X .
$$

Recall that every $U \in \mathcal{C}_{o v}(X)$ admits a $U^{\prime} \in \mathcal{C}_{o v}(X)$ such that $s \mathcal{U}^{\prime}=\left\{s t\left(U, U^{\prime}\right) \mid\right.$ $\left.U^{\prime} \in \mathcal{U}^{\prime}\right\}$ belongs to $\mathcal{C}_{o v}(X)$ and $s t U^{\prime}<\mathcal{U}$. We inductively define $s t^{0} \mathcal{U}=\mathcal{U}$, $s t^{1} \mathcal{U}$ ${ }_{s t} \mathcal{U}, \cdots, s^{n} \mathcal{U}=\left\{s t\left(U^{\prime}, \mathcal{U}\right) \mid U^{\prime} \in s^{n-1} t^{n}\right\}, n \in N$, which all belong to $\operatorname{Cov}(X)$. The above definition may be written as $s t^{n} U=s t\left(s t^{n-1} U, U\right)$. (Somewhere $s t^{n} \mathcal{U}$ is defined by $s t^{n} \mathcal{U}={ }_{s t}\left(s t^{n-1} \mathcal{U}\right)$ which only technically differs from the above).

(1.2) Definition. A (gauged) approximate system $\mathscr{X}$ is called uniform 
provided condition

$$
\mathcal{U}_{a^{\prime}}<p_{a a^{\prime}}^{-1} \mathcal{U}, \quad a<a^{\prime},
$$

is satisfied.

(1.3) Definition. A (gauged) approximate map $\boldsymbol{q}$ from a space $Y$ into a (gauged) approximate sytem $\mathscr{X}, \boldsymbol{q}: Y \rightarrow \mathfrak{X}$, is any collection $\boldsymbol{q}=\left\{q_{a} \mid a \in A\right\}=\left(q_{a}\right)$ of mappings $q_{a}: Y \rightarrow X_{a}$ such that

(AS) For every $a \in A$ and every $U \in \mathcal{C}_{o v}\left(X_{a}\right)$ there exists an $a^{\prime}>a$ such that $\left(q_{a}, p_{a a^{\prime \prime}} q_{a^{\prime \prime}}\right)<\mathcal{U}$, whenever $a^{\prime \prime}>a^{\prime}$.

(1.4) REMARK. Observe that in condition (AS) meshes do not appear. Therefore, a more appropriate condition for a gauged approximate map $\boldsymbol{q}: Y \rightarrow \mathfrak{X}$ should be

$$
\left(q_{a}, p_{a a^{\prime}} q_{a^{\prime}}\right)<{ }_{\mathrm{s}} \mathcal{U}_{a}, \quad a<a^{\prime} .
$$

(Of course, the above condition and (A2), (A3) of $\mathscr{X}$ imply (AS) for q.)

(1.5) Definition. A (gauged) approximate map $\boldsymbol{p}=\left(p_{a}\right): X \rightarrow \mathscr{X}$ is called a limit of $\mathscr{X}$ provided it has the following universal property:

(UL) For any approximate map $\boldsymbol{q}: Y \rightarrow \mathfrak{X}$ there exists a unique mapping $g: Y \rightarrow X$ satisfying $p_{a} g=q_{a}$, for every $a \in A$.

Since a limit space $X$ is determined up to a unique homeomorphism, we often speak of the limit $X$ of $\mathscr{X}$ and we write $X=\lim \mathscr{X}$.

We adopt Theorem (2.8) from [7] as the definition of a gauged approximate resolution of a space.

(1.6) Definition. A gauged approximate resolution of a space $X$ is any approximate map $\boldsymbol{p}: X \rightarrow \mathscr{X}$ satisfying the following two conditions :

(B1) $\quad(\forall U \in \operatorname{Cov}(X)) \quad(\exists a \in A) \quad\left(\forall a^{\prime}<a\right) \quad p_{a^{\prime}}^{-1} \bigcup_{a^{\prime}}<\mathscr{U}$;

(B2) $\quad(\forall a \in A) \quad\left(\exists a^{\prime}>a\right) \quad\left(\forall a^{\prime \prime}>a^{\prime}\right) \quad p_{a a^{\prime \prime}}\left(X_{a^{\prime \prime}}\right) \subseteq s t\left(p_{a}(X), \mathcal{U}_{a}\right)$.

A gauged approximate system $\mathscr{X}$ is said to be a gauged approximate resolution provided, there exist a topologically complete space $X$ and a gauged approximate resolution $\boldsymbol{p}: X \rightarrow \mathfrak{X}$ of $X$.

(1.7) Definition. A gauged approximate mapping $\boldsymbol{f}$ from a gauged approximate system $\mathscr{X}$ to a gauged approximate system $\mathscr{Y}=\left(Y_{b}, \mathcal{V}_{b}, q_{b b^{\prime}}, B\right), \boldsymbol{f}: \mathfrak{X} \rightarrow a$, is any collection $\boldsymbol{f}=\left\{f, f_{b} \mid b \in B\right\}$ consisting of a function $f: B \rightarrow A$ and of map- 
pings $f_{b}: X_{f(b)} \rightarrow Y_{b}, b \in B$, such that the following condition holds:

$(\mathrm{AM})$

$$
\begin{gathered}
\left(\forall b<b^{\prime} \text { in } B\right) \quad\left(\exists a>f(b), f\left(b^{\prime}\right) \text { in } A\right) \quad\left(\forall a^{\prime}>a\right) \\
\left(q_{b b^{\prime}} f_{b^{\prime}} p_{\left.f\left(b^{\prime}\right), a^{\prime}, f_{b} p_{f(b), a^{\prime}}\right)<s t \subset V_{b} .}\right.
\end{gathered}
$$

The set of all gauged approximate mappings from $\mathscr{X}$ to $\mathscr{G}$ is denoted by $\operatorname{AP}(\mathscr{X}, \mathscr{y})$.

A gauged approximate mapping $\boldsymbol{f}: \mathscr{X} \rightarrow \mathscr{y}$ is called uniform, provided $\boldsymbol{f}$ satisfies the additional condition

(AMU) $\quad \mathcal{U}_{f(b)}<f_{b}^{-1}\left(\mathcal{V}_{b}\right), \quad b \in B$.

(1.8) Definition. Let $\boldsymbol{f}, \boldsymbol{f}^{\prime}=\left\{f^{\prime}, f_{b}^{\prime} \mid b \in B\right\}: \mathscr{X} \rightarrow \mathscr{q}$ be gauged approximate mappings. We say that $\boldsymbol{f}$ is contiguous (or simply equivalent) to $\boldsymbol{f}^{\prime}, \boldsymbol{f} \equiv \boldsymbol{f}^{\prime}$, provided, for each $b \in B$, there exists an $a \in A, a>f(b), f^{\prime}(b)$, such that

(EM) $\quad\left(f_{b} p_{f(b), a^{\prime}}, f_{b}^{\prime} p_{f^{\prime}(b), a^{\prime}}\right)<{ }_{s} c \mathcal{V}_{b}$, whenever $a^{\prime}>a$.

The relation $\equiv$ generates an equivalence relation $\sim$ on the set $\operatorname{AP}(\mathscr{X}, \mathscr{q})$. The quotient set $\operatorname{AP}(\mathscr{X}, \mathscr{Y}) / \sim$ will be denoted by $\operatorname{AP}[\mathscr{X}, \mathscr{y}]$, and the equivalence class of $\boldsymbol{f}$ by $[\boldsymbol{f}]$.

If $\boldsymbol{f}: \mathcal{X} \rightarrow \mathscr{q}$ and $\boldsymbol{g}=\left\{g, g_{c} \mid c \in C\right\}: \mathscr{Y} \rightarrow \mathcal{Z}=\left(Z_{c}, W_{c}, r_{c c^{\prime}}, C\right)$ are gauged approximate maps, where $\boldsymbol{g}$ is uniform, then the collection $\left\{g f, g_{c} f_{\boldsymbol{g}(c)} \mid c \in C\right\}$ determines the "composite" gauged approximate map $g f: \mathscr{X} \rightarrow s t \mathcal{Z}^{*}=\left(Z_{c}, s t \mathscr{W}, r_{c c^{\prime}}, C^{*}\right)$, where $\mathscr{Z}^{*}=\left(Z_{c}, \mathscr{W}_{c}, r_{c c^{\prime}}, C^{*}\right)$ is the uniform system associated with $\mathscr{L}([7],(1.6)$ and (8.1)). By [7] (8.7), for any $\boldsymbol{f}: \mathscr{X} \rightarrow \mathscr{y}$ and $g: q] \rightarrow \mathscr{Z}$, where $C$ is cofinite, there exists a uniform $\boldsymbol{g}^{\prime}: \mathscr{y} \rightarrow \mathscr{Z}$ such that $\boldsymbol{g}^{\prime} \sim \boldsymbol{g}$ and $\boldsymbol{g}^{\prime} \boldsymbol{f}: \mathfrak{X} \rightarrow \mathscr{L}$.

(1.9) The category APRES. The class of objects $O b$ (APRES) is formed by all cofinite gauged approximate resolutions $\mathscr{X}$ consisting of topologically complete spaces. The set of morphisms $\operatorname{APRES}\left(\mathscr{X}, y_{y}\right)$ is the set $\operatorname{AP}[\mathscr{X}, \mathscr{y}]$. The identity morphism $1_{\mathscr{X}}$ on $\mathscr{X}$ is the class $\left[1_{\mathscr{X}}\right]$, where $1_{\mathscr{X}}=\left\{1_{\Lambda}, 1_{X_{a}} \mid a \in A\right\} \in$ $\operatorname{AP}(\mathscr{X}, \mathfrak{X})$. The existence of uniform representatives of morphisms and good properties of the limit guarantee the existence of a well-defined composition in APRES as well as its associativity ([7] (8.10), (8.11), (8.12)).

We finally mention the criterion for isomorphisms in APRES obtained in [8] (2.2).

(1.10) Let $\mathscr{X}, \mathscr{y} \in O b(\mathrm{APRES})$ and $\boldsymbol{f} \in \mathrm{AP}(\mathscr{X}, \mathscr{Y})$ be given. Then $[\boldsymbol{f}]$ is an isomorphism in APRES if and only if there exists an $n \in \boldsymbol{N}_{0}$ such that, for every $b \in B$, there is an $a_{0} \in A, a_{0}>f(b)$, such that, for any $a \in A$, there are a $b^{\prime}>b$, 
an $a^{\prime}>a, f\left(b^{\prime}\right)$ and a mapping $k: Y_{b^{\prime}} \rightarrow X_{a}$ satisfying the following three conditions :

(AD) $\quad\left(f_{b} p_{f(b), a} k q_{b^{\prime} b^{\prime \prime}}, q_{b b^{\prime \prime}}\right)<s t^{n} C V_{b}$, whənever $b^{\prime \prime}>b^{\prime}$;

(DA) $\quad\left(p_{a a^{\prime \prime}}, k f_{b^{\prime}} p_{f\left(b^{\prime}\right), a^{\prime \prime}}\right)<s^{n} q_{a}$, whenever $a^{\prime \prime}>a^{\prime}$;

(MU) $\quad \mathscr{V}_{b^{\prime}}<k^{-1}\left[U_{a}\right]^{\cdot}$

We conclude this introduction with the definition of a non-gauged approximate inverse system (approximate resolution) as in [4], adding several nontrivial examples of such systems.

(1.11) Definition. An approximate (inverse) system $\boldsymbol{X}$ is a collection $\left(X_{a}, p_{a a^{\prime}}, A\right)$ consisting of :

-a preordered set $A=(A,<)$, which is directed and unbounded;

-for each $a \in A$, a (topological) space $X_{a}$;

-for every related pair $a<a^{\prime}$, a (continuous) mapping $p_{a a^{\prime}}: X_{a^{\prime}} \rightarrow X_{a}\left(p_{a a}\right.$ $=1_{X_{a}}$ is the identity mapping on $X_{a}$ ). Moreover, the following condition is required :
(A) $\quad(\forall a \in A) \quad\left(\forall U \in \operatorname{Cov}\left(X_{a}\right)\right) \quad\left(\exists a_{0}>a\right) \quad\left(\forall a^{\prime \prime}>a^{\prime}>a_{0}\right)$
$\left(p_{a a^{\prime \prime}}, p_{a a^{\prime}} p_{a^{\prime} a^{\prime \prime}}\right)<U$.

Note that $(\mathrm{A})=(\mathrm{A} 2)$. The boldface characters denote approximate systems in the sense of the above definition, while gauged approximate systems are denoted by script characters.

(1.12) Definition. An approximate resolution of a space $X$ is any approximate map $\boldsymbol{p}=\left(p_{a}\right): X \rightarrow X=\left(X_{a}, p_{a a^{\prime}}, A\right)$ satisfying the following two conditions :

(R1) $\quad(\forall P \in \mathrm{POL}) \quad(\forall C V \in \mathcal{C}$ ov $(P)) \quad(\forall f: X \rightarrow P) \quad(\exists a \in A) \quad\left(\forall a^{\prime}>a\right)$

$$
\left(\exists g: X_{a^{\prime}} \rightarrow P\right) \quad\left(g p_{a^{\prime}}, f\right)<\mathcal{C} \text {; }
$$

(R2) $(\forall P \in \mathrm{POL})\left(\forall C V \in \mathcal{C}_{o v}(P)\right)\left(\exists C \mathcal{V}^{\prime} \in \mathcal{C}_{o v}(P)\right)(\forall a \in A)\left(\forall g, g^{\prime}: X_{a} \rightarrow P\right)$

$$
\left(g p_{a}, g^{\prime} p_{a}\right)<\mathcal{V}^{\prime} \Rightarrow\left(\left(\exists a^{\prime}>a\right)\left(\forall a^{\prime \prime}>a^{\prime}\right)\left(g p_{a a^{\prime \prime}}, g^{\prime} p_{a a^{\prime \prime}}\right)<\mathcal{Q}\right),
$$

where POL denotes the class of all polyhedra (CW-topology).

An approximate system $\boldsymbol{X}$ is said to be an approximate resolution provided, there exist a topologically complete space $X$ and an approximate resolution $\boldsymbol{p}: X \rightarrow \boldsymbol{X}$ of $X$.

(1.13) Trivial Examples. (a) Every usual (commutative) inverse system 
(resolution of a space $X,[3]$ ) is an approximate system (approximate resolution of $X$ ). (This is not true neither for gauged approximate systems nor for gauged approximate resolutions; see (1.14) below).

(b) Every gauged approximate system $\mathscr{X}$ (gauged approximate resolution $\boldsymbol{p}: X \rightarrow \mathfrak{X}$ of $X$ ), after forgetting of the meshes, becomes an approximate system $\boldsymbol{X}$ (approximate resolution $\boldsymbol{p}: X \rightarrow X$ of $X$ ).

(c) Every approximate system (approximate resolution) $\boldsymbol{X}$, which admits gauges in the sense of $[7](1.7)([7](3.4))$, is an approximate system (approximate resolution).

(1.14) REMARK AND NONTRIVIAL EXAMPLES. One naturally asks if it is possible to choose coverings $\mathcal{U}_{a} \in \mathcal{C}_{o v}\left(X_{a}\right)$ such that a given approximate system $\boldsymbol{X}=\left(X_{a}, p_{a a^{\prime}}, A\right)$ becomes a gauged approximate system $\mathscr{X}=\left(X_{a}, \mathcal{U}_{a}, p_{a a^{\prime}}, A\right)$ ? If $\boldsymbol{X}$ is commutative, all $X_{a}$ are compact metric and $A$ is cofinite, the answer is affirmative $([9](3.8),[7](1.8))$. The same holds even without assuming commutativity. However, the preorder of $A$ has to be then slightly changed to preserve condition (A1).

The following examples show that the answer to the above question is negative even in the commutative case of compact Hausdorff or locally compact metric (polyhedral) terms.

Let $\tau$ be an uncountable cardinal number and let $I^{\tau}$ be the corresponding Tychonoff cube. Then $I^{\tau}$ is compact Hausdorff but not metrizable. Take $\boldsymbol{X}=$ $\left(X_{n}, p_{n n^{\prime}}, \boldsymbol{N}\right)$, where $X_{n}=I^{\tau}$, for all $n \in \boldsymbol{N}$, and $p_{n n^{\prime}}=1_{I \tau}$, for all pairs $n \leqq n^{\prime}$. Then $\boldsymbol{X}$ is a usual (commutative) inverse sequence, and thus, an approximate system. Suppose that there exists a sequence of normal coverings $\bigcup_{n} \in \mathcal{C}_{o v}\left(X_{n}\right)$, $n \in \boldsymbol{N}$, such that $\mathscr{X}=\left(X_{n}, \mathcal{U}_{n}, p_{n n^{\prime}}, \boldsymbol{N}\right)$ is a gauged approximate system. Then (A3) implies that $\left\{U_{n} \mid n \in \boldsymbol{N}\right\}$ is a cofinal subfamily of $\operatorname{Cov}\left(I^{\tau}\right)$.

ClaIm. $\left\{U_{n} \mid n \in \boldsymbol{N}\right\}$ is a development of $I^{\tau}$, i.e., (see [2], p. 408), for every $x \in I^{\tau}$ and every neighbourhood $V_{0}(x)$ in $I^{\tau}$ there exists an $n \in \boldsymbol{N}$ such that $\operatorname{st}\left(x, \mathcal{V}_{n}\right) \subseteq V_{0}$. Indeed, let $x \in I^{\tau}$ and let $V_{0}$ be any neighbourhood of $x$ in $I^{\tau}$. Since $I^{\tau}$ is regular, there exists a neighbourhood $U$ of $x$ such that $U \subseteq \bar{U}$ $\cong V_{0}$. Take $V_{1}=I^{\tau} \backslash \bar{U}$. Then $\mathcal{Q}=\left\{V_{0}, V_{1}\right\} \in \mathcal{C}_{o v}\left(I^{\tau}\right)$. Choose a $c V^{\prime} \in \mathcal{C}_{o v}\left(I^{\tau}\right)$ satisfying ${ }_{t} c V^{\prime}<\mathcal{V}$. Then there exists a $V^{\prime} \in \mathcal{C V}^{\prime}$ such that $x \in V^{\prime}$ and $s t\left(V^{\prime}, C V^{\prime}\right)$ $\subseteq V_{0}$. Since $\left\{\mathcal{U}_{n} \mid n \in N\right\}$ is cofinal in $\mathcal{C}_{o v}\left(I^{\tau}\right)$, there is an $n \in N$ such that $\mathcal{U}_{n}<\mathcal{C V}^{\prime}$. Consequently,

$$
s t\left(x, \mathcal{U}_{n}\right) \cong s t\left(x, \mathcal{V}^{\prime}\right) \subseteq s t\left(V^{\prime}, \mathcal{V}^{\prime}\right) \cong V_{0}
$$

and the claim is proved. 
Now, since $I^{\tau}$ is collectionwise normal, the Bing metrization theorem $([2]$ (5.4.1)) implies metrizability of $I^{\tau}$, which is a contradiction. Therefore, $X$ does not admit gauges.

In the same way, using collectionwise normal non-metrizable spaces, one can construct inverse sequences which do not admit gauges.

Another example in the (commutative) locally compact metric case is $\boldsymbol{X}=$ $\left(X_{n}, p_{n n^{\prime}}, \boldsymbol{N}\right)$, where $X_{n}=[0,1) \leqq \boldsymbol{R}$, for every $n$, and $p_{n n^{\prime}}$ is the identity mapping for every pair $n \leqq n^{\prime}$. Since $\operatorname{Cov}([0,1))$ has no countable cofinal subfamily (compare [6], Examples 4 and 5), again (A3) cannot be fulfilled for any choice of $\mathcal{U}_{n} \in \operatorname{Cov}\left(X_{n}\right), n \in \boldsymbol{N}$.

\section{The main construction}

There are various ways how to associate a gauged approximate system with a non-gauged one. A very general construction of that kind was exhibited by S. Mardešić in [4]. However, its main properties ([4], Theorem 1) can also be obtained by adapting a much simpler construction due to $T$. Watanabe [9] $(3.7))$.

(2.1) Let $X=\left(X_{a}, p_{a a^{\prime}}, A\right)$ be an approximate system. For each $a \in A$ choose a cofinal subfamily $\mathcal{C}_{a} \subseteq \mathcal{C}_{o v}\left(X_{a}\right)$ of the minimal cardinality. Consider the family $\mathcal{A}=\left\{(a, \mathcal{U}) \mid a \in A, \mathcal{V} \in \mathcal{C}_{a}\right\}=\bigcup_{a \in A}\left(\{a\} \times \mathcal{C}_{a}\right)$. Define $\Lambda=\{\lambda \subseteq \mathcal{A} \mid \varnothing \neq \lambda$ finite and order it by inclusion. Then $\Lambda$ is a directed, unbounded, cofinite and antisymmetric set. Therefore, there exists an increasing surjection $s: \Lambda \rightarrow A$ such that $s(\{(a, U)\})=a$, for every $(a, \mathcal{U}) \in \mathcal{A}$. For each $\lambda \in \Lambda$, define

$$
X_{\lambda}^{*}=X_{s(\lambda)} \text { and } U_{\lambda}^{*}=\bigwedge_{i=1}^{n} p_{a_{i}, s(\lambda)}^{-1} \mathcal{U}_{i} \in \mathcal{C} \text { ov }\left(X_{\lambda}^{*}\right),
$$

whenever $\lambda=\left\{\left(a_{1}, V_{1}\right), \cdots,\left(a_{n}, V_{n}\right)\right\}$. Since $s$ increases, the covering $U_{\lambda}^{*}$ is well defined. Observe that $\mathcal{U}_{(a, \mathcal{U})}^{*}=\mathcal{U}$, for any $(a, \mathcal{U}) \in \mathcal{A}$. For every pair $\lambda<\lambda^{\prime}$ in $\Lambda$, put

$$
p_{\lambda \lambda^{\prime}}^{*}=p_{s(\lambda), s\left(\lambda^{\prime}\right)}: X_{\lambda^{\prime}}^{*} \rightarrow X_{\Lambda}^{*} .
$$

Let us show that the collection $\left(X_{\lambda}^{*}, U_{\lambda}^{*}, p_{\lambda \lambda^{\prime}}^{*}, \Lambda\right)$ satisfies conditions (A2) and (A3) of Definition (1.1).

(A2). Let $\lambda \in \Lambda$ and $U \in \mathcal{C}_{o v}\left(X_{\lambda}^{*}=X_{s(\lambda)}\right)$ be given. For $s(\lambda)$ and $U$, choose an $a_{0}>s(\lambda)$ by (A) of $X$. Since $s$ is an increasing surjection, there exists a $\lambda^{\prime}>\lambda$ such that $s\left(\lambda^{\prime}\right)>a_{0}$. If $\lambda_{2}>\lambda_{1}>\lambda^{\prime}$, then $s\left(\lambda_{2}\right)>s\left(\lambda_{1}\right)>s\left(\lambda^{\prime}\right)>a_{0}$ and therefore,

$$
p_{\lambda \lambda_{2}}^{*}=p_{s(\lambda), s\left(\lambda_{2}\right)}=q p_{s(\lambda), s\left(\lambda_{1}\right)} p_{s\left(\lambda_{1}\right), s\left(\lambda_{2}\right)}=p_{\lambda_{1} \lambda_{1}}^{*} p_{\lambda_{1} \lambda_{2}}^{*} .
$$


(A3). Let $\lambda \in \Lambda, \lambda=\left\{\left(a_{1}, \mathcal{U}_{1}\right), \cdots,\left(a_{m}, \mathcal{U}_{m}\right)\right\}$, and let $U \in \mathcal{C}_{o v}\left(X_{\lambda}^{*}=X_{s(\lambda)}\right)$ be given. Take a $U^{\prime} \in \mathcal{C}_{s(\lambda)}$ refining $\mathscr{U}$ and let $\lambda^{\prime}=\lambda \cup\left\{\left(s(\lambda), U^{\prime}\right)\right\}$. Then $\lambda^{\prime}>\lambda$. If $\lambda^{\prime \prime}>\lambda^{\prime}$, i.e., $\lambda^{\prime \prime}=\left\{\left(a_{1}, U_{1}\right), \cdots,\left(a_{m}, \mathcal{U}_{m}\right),\left(s(\lambda), \mathcal{U}^{\prime}\right),\left(a_{m+1}, U_{m+1}\right), \cdots,\left(a_{n}, U_{n}\right)\right\}$, then

$$
\begin{aligned}
U_{\lambda^{\prime \prime}}^{*} & =\left(\bigwedge_{i=1}^{n} p_{a}^{-1}{ }_{i, s\left(\lambda \lambda^{\prime \prime}\right)} U_{i}\right) \wedge p_{s(\lambda), 8\left(\lambda \lambda^{\prime \prime}\right)}^{-1} U^{\prime}<p_{s(\lambda) s\left(\lambda \lambda^{\prime \prime}\right)}^{-1} U^{\prime} \\
& =p_{\lambda \lambda^{\prime \prime}}^{*-1} U^{\prime}<p_{\lambda \lambda^{\prime \prime}}^{*-1} U .
\end{aligned}
$$

(2.2) Let us define a new relation $<^{*}$ on $\Lambda$ by putting $\lambda<* \lambda^{\prime}$ provided $\lambda=\lambda^{\prime}$, or $\lambda \neq \lambda^{\prime}, \lambda<\lambda^{\prime}$ and $\left(\forall \lambda_{2}>\lambda_{1}>\lambda^{\prime}\right)$

$$
\left(p \lambda_{\lambda_{2}}^{*}, p_{\lambda \lambda_{1}}^{*} p_{\lambda_{1} \lambda_{2}}^{*}\right)<U_{\lambda}^{*}, \quad U_{\lambda_{1}}^{*}<\left(p_{\lambda \lambda_{1}}^{*}\right)^{-1} \mathcal{U}_{\lambda}^{*} .
$$

Note that $\lambda<* \lambda^{\prime}$ implies $\lambda<\lambda^{\prime}$, and $\lambda<* \lambda^{\prime}<\lambda^{\prime \prime}$ and $\lambda \neq \lambda^{\prime}$ imply $\lambda<* \lambda^{\prime \prime}$. Since the collection ( $\left.X_{\Lambda}^{*}, U^{*}, p_{\lambda \lambda^{\prime}}^{*}, \Lambda\right)$ satisfies (A2) and (A3), $\Lambda^{*}=\left(\Lambda,<^{*}\right)$ is a directed, unbounded, cofinite and antisymmetric set. Furthermore, the collection $\left(X_{\lambda}^{*}, U_{\lambda}^{*}\right.$, $p_{\wedge \lambda^{\prime}}^{*}, \Lambda^{*}$ ) also satisfies conditions (A2) and (A3). Moreover, it obviously satisfies (A1) and (AU) too. Therefore, $X^{*}=\left(X_{\Lambda}^{*}, U_{\lambda}^{*}, p_{\lambda^{\prime}}^{*}, \Lambda^{*}\right)$ is a uniform gauged approximate system. Hence, we have proved the following theorem:

(2.3) THEOREM. For every approximate system $X=\left(X_{a}, p_{a a^{\prime}}, A\right)$ there exist a gauged approximate system $\mathfrak{X}^{*}=\left(X_{\lambda}^{*}, \mathcal{U}_{\lambda}^{*}, p_{\lambda^{\prime}}^{*}, \Lambda^{*}\right)$ and a function $s: \Lambda^{*} \rightarrow A$ having the following properties:

(i) $\Lambda^{*}$ is cofinite and antisymmetric;

(ii) $s$ is increasing and surjective;

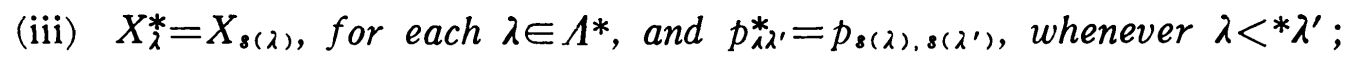

(iv) for any $a \in A$ and $U \in \mathcal{C}_{o v}\left(X_{a}\right)$, there is $a \lambda \in \Lambda^{*}$ such that $s(\lambda)=a$ and $U_{\lambda}^{*}<U$

(v) $\mathfrak{X}^{*}$ is uniform.

A gauged system $\mathfrak{X}^{*}$ with properties $(\mathrm{i})-(\mathrm{v})$ is said to be induced by $\boldsymbol{X}$.

(2.4) REMARK AND QUESTION. In the construction of a gauged approximate system $\mathcal{X}$, associated with a given approximate system $\boldsymbol{X}$, it is often convenient to obtain $\mathscr{X}$ with an indexing set of minimal cardinality. In the preceding construction the entire family $\mathcal{A}^{\prime}=\bigcup_{a \in A}\left(\{a\} \times \mathcal{C}_{o v}\left(X_{a}\right)\right)$ would also do. However, $\mathfrak{X}^{*^{\prime}}$, obtained in that way, would have $\Lambda^{*^{\prime}}$ as its indexing set and this set is, in general, of a larger cardinality than $\Lambda^{*}$. Moreover, $\Lambda^{*}$ even need not be cofinal in $\Lambda^{*^{\prime}}$. Of course, the construction in (2.1) may also start with any cofinal $A^{\prime} \cong A$. 
The above consideration raises the following question: Let $\boldsymbol{X}=\left(X_{a}, p_{a a^{\prime}}, A\right)$ be an approximate system which does not admit gauges, and let $\mathscr{X}=\left(X_{b}^{\prime}, \mathcal{U}_{b}\right.$, $\left.p_{b b^{\prime}}^{\prime}, B\right)$ be a gauged approximate system such that each $X_{b}^{\prime}$ is an $X_{a}$ and each $p_{b b^{\prime}}^{\prime}$ is a $p_{a a^{\prime}}$. Is it necessarily $|B| \geqq\left|A^{*}\right|(=|A|)$ ? (Assume that $A$ is obtained via a cofinal $A^{\prime} \subseteq A$ and a cofinal $\mathcal{C}_{a} \subseteq \mathcal{C}_{o v}\left(X_{a}\right), a \in A^{\prime}$, all of minimal cardinalities!)

We now center our attention to the relation between a given approximate map $\boldsymbol{q}: Y \rightarrow X$ and the induced (gauged) approximate map $\boldsymbol{q}^{*}: Y \rightarrow \mathfrak{X}^{*}$, and vice versa.

Let $\boldsymbol{q}=\left(q_{a}\right): Y \rightarrow \boldsymbol{X}$ be an approximate map from a space $Y$ into an approximate system $\boldsymbol{X}$. Define the collection $\boldsymbol{q}^{*}=\left(q_{\lambda}^{*}\right)$, by putting $q_{\lambda}^{*}=q_{\boldsymbol{s}(\lambda)}: Y \rightarrow X_{\boldsymbol{s}(\lambda)}$ $=X_{\lambda}^{*}, \lambda \in \Lambda^{*}$. Conversely, if a gauged approximate map $\boldsymbol{q}=\left(q_{\lambda}\right): Y \rightarrow \mathfrak{X}^{*}$ is given, define the collection $\overline{\boldsymbol{q}}=\left(\bar{q}_{a}\right)$, by putting $\bar{q}_{a}=q_{\sigma(a)}: Y \rightarrow X_{\sigma(a)}^{*}=X_{a}, a \in A$, where $\sigma: A \rightarrow \Lambda^{*}$ is a section of $s$, i.e., $s \sigma=1_{A}$.

We say that $\boldsymbol{q}^{*}$ is induced by $\boldsymbol{q}$ and that $\overline{\boldsymbol{q}}$ is associated with $\boldsymbol{q}$.

Observe that $\left(\boldsymbol{q}^{*}\right)^{-}=\boldsymbol{q}: Y \rightarrow Y$, but in general $(\overline{\boldsymbol{q}})^{*} \neq \boldsymbol{q}: Y \rightarrow \mathfrak{X}^{*}$ (see (2.6) below).

As in [4], one can easily prove:

(2.5) (i) If $\boldsymbol{q}: Y \rightarrow Y$ is an approximate map, then the induced $\boldsymbol{q}^{*}: Y \rightarrow \mathscr{X}$ is a gauged approximate map.

(ii) If $\boldsymbol{q}: Y \rightarrow \mathfrak{X}^{*}$ is a gauged approximate map, then any associated $\overline{\boldsymbol{q}}: Y \rightarrow \mathfrak{X}$ is an approximate map.

Since Theorem (2.3) in essence coincides with [4], Theorem 1, all the corresponding statements from [4] may be transferred to our $\mathfrak{X}^{*}, \boldsymbol{q}^{*}: Y \rightarrow \mathfrak{X}^{*}$ and $\overline{\boldsymbol{q}}: Y \rightarrow \boldsymbol{X}$. Therefore, we shall not repeat them. Only the following three facts are not to be found in [4].

(2.6) Lemma. Let a gauged approximate map $\boldsymbol{q}: Y \rightarrow \mathfrak{X}^{*}$ be given. If in the approximate system $\boldsymbol{X}$ all $X_{a}$ are Tychonoff spaces, then $(\overline{\boldsymbol{q}})^{*}=\boldsymbol{q}$, for any associated $\overline{\boldsymbol{q}}: Y \rightarrow \boldsymbol{X}$.

ProOF. Note that $(\overline{\boldsymbol{q}})^{*}=\left(q_{\sigma s(\lambda)}\right): Y \rightarrow \mathfrak{X}^{*}$, where $\sigma$ is a section of $s$. We must prove that $q_{\sigma s(\lambda)}=q_{\lambda}, \lambda \in \Lambda^{*}$. Since $X_{\sigma s(\lambda)}^{*}=X_{s(\lambda)}=X_{\lambda}^{*}, \lambda \in \Lambda^{*}$, and all $X_{a}$ are Tychonoff spaces, it is sufficient to verify the following:

$$
\left(\forall \lambda \in \Lambda^{*}\right) \quad\left(\forall U \in \mathcal{C}_{o v}\left(X_{s(\lambda)}\right)\right) \quad\left(q_{\sigma s(\lambda)}, q_{\lambda}\right)<U .
$$

Indeed, by (AS) of $\boldsymbol{q}$ for $\lambda, \sigma s(\lambda)$ and a $U^{\prime},{ }_{s} U^{\prime}<U$, there exists a $\lambda^{\prime}>* \lambda, \sigma s(\lambda)$ such that 


$$
\begin{aligned}
& \left(q_{\lambda}, p_{\lambda^{\prime} \lambda^{\prime}}^{*} q_{\lambda^{\prime}}\right)<\mathcal{U}^{\prime} \\
& \left(q_{\sigma s(\lambda)}, p_{\sigma s(\lambda), \lambda}^{*}, q_{\lambda^{\prime}}\right)<\mathcal{U}^{\prime} .
\end{aligned}
$$

Hence,

$$
q_{\sigma s(\lambda)}=q^{\prime} p_{\sigma s(\lambda), \lambda^{\prime}}^{*}, q_{\lambda^{\prime}}=p_{s(\lambda), s\left(\lambda^{\prime}\right)}, q_{\lambda^{\prime}}=p_{\lambda, \lambda^{\prime}}^{*}, q_{\lambda^{\prime}}=q^{\prime} q_{\lambda}
$$

and consequently

$$
\left(q_{\sigma s(\lambda)}, q_{\lambda}\right)<s t \mathcal{U}^{\prime}<\mathcal{U} .
$$

(2.7) Let in an approximate system $X$ all $X_{a}$ be Tychonoff spaces and let $\boldsymbol{p}=\left(p_{\lambda}\right): X \rightarrow \mathscr{X}^{*}$ be an approxımate map. Then $\boldsymbol{p}$ is a limit if and only if any $\overline{\boldsymbol{p}}=\left(\bar{p}_{a}=p_{\sigma(a)}\right): X \rightarrow \boldsymbol{X}$ is a limit.

Proof. Since all $X_{a}$ from $X$ are Tychonoff spaces we may apply the analogue of [4], Theorem 4 and our Lemma (2.6). Hence, $\overline{\boldsymbol{p}}$ is a limit if and only if $(\bar{p})^{*}=\boldsymbol{p}$ is a limit.

In an analogous way one obtaines

(2.8) Let in an approximate system $X$ all $X_{a}$ be Tychonoff spaces and let $\boldsymbol{p}=\left(p_{\lambda}\right): X \rightarrow X^{*}$ be an approximate map. Then $\boldsymbol{p}$ is a gauged approximate resolution of $X$ if and only if any $\overline{\boldsymbol{p}}=\left(\bar{p}_{a}=p_{\sigma(a)}\right): X \rightarrow \boldsymbol{X}$ is an approximate resolution of $X$.

\section{The uniqueness of $\mathfrak{X}^{*}$}

The theorem below indicates which of the properties (2.3), (i)-(v), are "universal", i.e., essential in the theory of (gauged) approximate resolutions. As a consequence, it assures the uniqueness (up to isomorphism) of the induced gauged approximate resolution.

(3.1) TheOREM. Let $X=\left(X_{a}, p_{a a^{\prime}}, A\right)$ be an approximate resolution consisting of topologically complete spaces. Let $\mathfrak{X}^{\prime}=\left(X_{b}^{\prime}, \mathcal{V}_{b}^{\prime}, p_{b b^{\prime}}^{\prime}, B\right), \mathscr{X}^{\prime \prime}=\left(X_{c}^{\prime \prime}, \mathcal{U}_{c}^{\prime \prime}\right.$, $\left.p_{c c^{\prime}}^{\prime \prime}, C\right)$ be gauged approximate resolutions and let $s^{\prime}: B \rightarrow A, s^{\prime \prime}: C \rightarrow A$ be functions having the following properties:

(i) $B$ and $C$ are cofinite;

(ii) $s^{\prime}$ and $s^{\prime \prime}$ are increasing and surjective;

(iii) $X_{b}^{\prime}=X_{s^{\prime}(b)}$, for each $b \in B, p_{b b^{\prime}}^{\prime}=p_{s^{\prime}(b), s^{\prime}\left(b^{\prime}\right)}$, whenever $b<b^{\prime}, X_{c}^{\prime \prime}=X_{8^{\prime \prime}(c)}$,

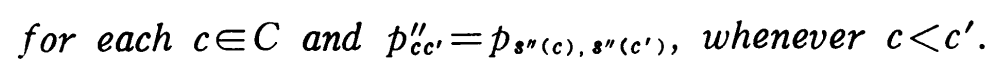

Then $\mathfrak{X}^{\prime}$ is isomorphic to $\mathfrak{X}^{\prime \prime}$ in the category APRES.

Proof. First of all, note that the assumption on $\boldsymbol{X}$ and property (i) guar- 
antee that $\mathscr{X}^{\prime}, \mathscr{X}^{\prime \prime} \in O b(\mathrm{APRES})$. Since $\mathscr{X}^{*}=\left(X_{k}^{*}, U_{\lambda}^{*}, p_{\lambda \lambda^{\prime}}^{*}, \Lambda^{*}\right)$ and $s: \Lambda^{*} \rightarrow A$ have all the above properties, if suffices to prove the theorem in the case $\mathscr{X}^{\prime \prime}$ $=X^{*}$. Let $u: \Lambda^{*} \rightarrow \Lambda^{*}$ be an increasing function defined by choosing a $u(\lambda)>*$ $>* \lambda$ such that $s u(\lambda)$ satisfies condition (A) of $\boldsymbol{X}$ simultaneously, for $s\left(\lambda_{1}\right), \cdots$, $s\left(\lambda_{k}\right), s(\lambda)$ and $U_{\lambda_{1}}^{*}, \cdots, U_{\lambda_{k}}^{*}, U_{\lambda}^{*}$ respectively, where $\lambda_{1}, \cdots, \lambda_{k}$ are all the predecessors of $\lambda$ in $\Lambda^{*}$ (such a function $u$ exists, since $\Lambda^{*}$ is cofinite and $s$ is increasing and surjective). Furthermore, since $s^{\prime}$ is surjective, there exists a function $g: \Lambda^{*} \rightarrow B$ such that $s^{\prime} g=s$. Then, $s u=s^{\prime} g u: \Lambda^{*} \rightarrow A$. Take $f=g u$ : $\Lambda^{* \rightarrow B}$ and

$$
f_{\lambda}=p_{\lambda, u(\lambda)}^{*} 1_{X_{s(\lambda)}}=p_{s(\lambda), s u(\lambda)}: X_{f(\lambda)}^{\prime}=X_{s u(\lambda)} \rightarrow X_{s(\lambda)}=X_{\Lambda}^{*}, \quad \lambda \in \Lambda^{*} .
$$

Then, $\boldsymbol{f}=\left\{f, f_{\lambda} \mid \lambda \in \Lambda^{*}\right\}: \mathscr{X}^{\prime} \rightarrow \mathfrak{X}^{*}$ is a gruged approximate map. Indeed, If any $\lambda<* \lambda^{\prime}$ in $\Lambda^{*}$ are given, choose a $b>f(\lambda), f\left(\lambda^{\prime}\right)$ in $B$ and let a $b^{\prime}>b$ be given. Then,

$$
\begin{aligned}
& p_{\lambda^{\prime} \lambda^{\prime}}^{*} f_{\lambda^{\prime}} p_{f\left(\lambda^{\prime}\right), b^{\prime}}^{\prime}=p_{\lambda^{\prime}}^{*} p_{\lambda^{\prime}}^{*}, u\left(\lambda^{\prime}\right) 1 p_{g u\left(\lambda^{\prime}\right), b^{\prime}}^{\prime} \\
& =p_{s(\lambda), s\left(\lambda^{\prime}\right)} p_{s\left(\lambda^{\prime}\right), s u\left(\lambda^{\prime}\right)} p_{s^{\prime} g u\left(\lambda^{\prime}\right), s^{\prime}\left(b^{\prime}\right)} \\
& =q_{1}^{*} p_{s(\lambda), s u\left(\lambda^{\prime}\right)} p_{s u\left(\lambda^{\prime}\right), s^{\prime}\left(b^{\prime}\right)}=q_{\Lambda}^{*} p_{s(\lambda), \delta^{\prime}\left(b^{\prime}\right)} \\
& =q_{\lambda}^{*} p_{s(\lambda), s u(\lambda)} p_{s u(\lambda), s^{\prime}\left(b^{\prime}\right)}=p_{\lambda, u(\lambda)}^{*} 1 p_{g}^{\prime} u(\lambda), b^{\prime} \\
& =f_{\lambda} p_{f}^{\prime}(\lambda), b^{\prime} \text {. }
\end{aligned}
$$

Therefore,

$$
\left(p_{\lambda \lambda^{\prime}}^{*} f_{\lambda^{\prime}} p_{f}^{\prime}\left(\lambda^{\prime}\right), b^{\prime}, f_{\lambda} p_{f}^{\prime}(\lambda), b^{\prime}\right)<s t U_{\lambda}^{*},
$$

which establishes condition (AM), for $f \in \mathrm{AP}\left(\mathfrak{X}^{\prime}, \mathfrak{X}^{*}\right)$. We now apply (1.10) to prove that $[\boldsymbol{f}]$ is an isomorphism in APRES. Take $n=1$. If $\lambda \in \Lambda^{*}$, take $b_{0}=$ $f(\lambda)$ and let any $b>b_{0}$ in $B$ be given. Pick up a $U \in \mathcal{C}_{s^{\prime}(b)}$ (see (2.1)) refining $\mathcal{U}_{b}^{\prime} \in \mathcal{C}_{o v}\left(X_{b}^{\prime}=X_{s^{\prime}(b)}\right)$ and then choose a $\lambda^{\prime}>* \lambda$ in $A^{*}$, such that $\left(s^{\prime}(b), U\right) \in \lambda^{\prime}$ and $s\left(\lambda^{\prime}\right)$ satisfies condition (A) of $X$, for $s^{\prime}(b)$ and $U_{b}^{\prime}$. Note that $s\left(\lambda^{\prime}\right)>s^{\prime}(b)>$ $s^{\prime}\left(b_{0}\right)=s^{\prime} f(\lambda)=s^{\prime} g u(\lambda)=s u(\lambda)$ in $A$. Choose a $b^{\prime}>b, f\left(\lambda^{\prime}\right)$ and take $k=p_{s^{\prime}(b), s\left(\lambda^{\prime}\right)}$ : $X_{\lambda^{\prime}}^{*}=X_{s\left(\lambda^{\prime}\right)} \rightarrow X_{s^{\prime}(b)}=X_{b}^{\prime}$. Let any $\lambda^{\prime \prime}>* \lambda^{\prime}$ and $b^{\prime \prime}>b^{\prime}$ be given. Then,

Hence,

$$
\begin{aligned}
& f_{\lambda} p_{f}^{\prime}(\lambda), b p_{\lambda^{\prime} \lambda^{\prime \prime}}=p_{s(\lambda), s u(\lambda)} p_{s u(\lambda), s^{\prime}(b)} p_{8^{\prime}(b), s\left(\lambda^{\prime}\right)} p_{s\left(\lambda^{\prime}\right), s\left(\lambda^{\prime \prime}\right)} \\
& =V_{\Lambda}^{*} p_{s(\lambda), s^{\prime}(b)} p_{s^{\prime}(b), 8\left(\lambda^{\prime}\right)} p_{s\left(\lambda^{\prime}\right), s\left(\lambda^{\prime \prime}\right)} \\
& =q_{\lambda}^{*} p_{s}(\lambda), s\left(\lambda^{\prime}\right) p_{s\left(\lambda^{\prime}\right), s\left(\lambda^{\prime \prime}\right)}=p_{\lambda \lambda^{\prime}}^{*} p_{\lambda^{\prime} \lambda^{\prime \prime}}^{*}=q_{\alpha}^{*} p_{\lambda \lambda^{\prime \prime}}^{*} .
\end{aligned}
$$

$$
\left(f_{\lambda} p_{f(\lambda), b}^{\prime} k p_{\lambda^{\prime} \lambda \lambda^{\prime \prime}}^{*}, p_{\lambda \lambda \lambda^{\prime \prime}}^{*}\right)<s t U_{\lambda}^{*},
$$

which verifies condition (AD) for $\boldsymbol{f}$. Furthermore, 
and consequently

$$
k f_{\lambda^{\prime}} p_{f\left(\lambda^{\prime}\right), b^{\prime \prime}}^{\prime}=p_{s^{\prime}(b), s\left(\lambda^{\prime}\right)} p_{s\left(\lambda^{\prime}\right), s u\left(\lambda^{\prime}\right)} p_{s u\left(\lambda^{\prime}\right), s^{\prime}\left(b^{\prime \prime}\right)}
$$

$$
=q_{b}^{\prime} p_{s^{\prime}(b), s u\left(\lambda^{\prime}\right)} p_{s u\left(\lambda^{\prime}\right), s^{\prime}\left(b^{\prime \prime}\right)}=q_{b}^{\prime} p_{s^{\prime}(b), s^{\prime}\left(b^{\prime \prime}\right)}=p_{b b^{\prime \prime}}^{\prime},
$$

$$
\left(k f_{\lambda^{\prime}} p_{f\left(\lambda^{\prime}\right), b^{\prime \prime}}^{\prime}, p_{b b_{u}}^{\prime}\right)<s t^{C} U_{b}^{\prime},
$$

which proves condition (DA) for $\boldsymbol{f}$. Finally, by (2.1),

$$
U_{\lambda^{\prime}}^{*}<\left(p_{s^{\prime}(b), s\left(\lambda^{\prime}\right)}\right)^{-1} U<\left(p_{s^{\prime}(b), s\left(\lambda^{\prime}\right)}\right)^{-1} U_{b}^{\prime}=k^{-1} U_{b}^{\prime},
$$

which verifies (MU) for $\boldsymbol{f}$. This completes the proof of the theorem.

As an immediate consequence of Theorems (3.1) and (2.3) we get the following corollary:

(3.2) COROLlARY. Let $\boldsymbol{X}=\left(X_{a}, p_{a a^{\prime}}, A\right)$ be an approximate resolution consisting of topologically complete spaces and let $\mathfrak{X}^{*}$ be its induced gauged approximate system.

(i) If $\mathfrak{X}^{*^{\prime}}$ is induced by $\boldsymbol{X}$ via any cofinal $A^{\prime} \cong A$ and $\mathcal{C}_{a}^{\prime} \cong \mathcal{C}_{o v}\left(X_{a}\right), a \in A^{\prime}$, and any increasing surjection $s^{\prime}: A^{* \prime} \rightarrow A^{\prime}$, then $\mathscr{X}^{* \prime} \cong X^{*}$ in APRES.

(ii) If $X$ is obtained by omittng the gauges of an $\mathscr{X}$ belonging to APRES, then $\mathscr{X} \cong \mathfrak{X}^{*}$ in APRES.

(iii) If $X^{*}$ is obtained by omitting the gauges of $\mathfrak{X}^{*}$ and if $\mathfrak{X}^{* *}$ is induced by $X^{*}$, then $\mathfrak{X}^{* *} \cong X^{*}$ in APRES.

\section{The induced gauged approximate mapping}

In this section we briefly sketch an extension of the *-construction $\mathscr{X} \mapsto \mathfrak{X}^{*}$ onto approximate mappings $\boldsymbol{f} \mapsto \boldsymbol{f}^{*}: \mathfrak{X}^{*} \rightarrow \mathfrak{y}^{*}$. First, we have to define a generalization of the notion of a gauged approximate mapping (1.7), suitable for the nongauged case. A natural one is the following

(4.1) Definition. An approximate mapping $\boldsymbol{f}$ from an approximate system $\boldsymbol{X}=\left(X_{a}, p_{a a^{\prime}}, A\right)$ to an approximate system $\boldsymbol{Y}=\left(Y_{b}, q_{b b^{\prime}}, B\right), \boldsymbol{f}: \boldsymbol{X} \rightarrow \boldsymbol{Y}$, is any collection $\boldsymbol{f}=\left\{f, f_{b} \mid b \in B\right\}$ consisting of a function $f: B \rightarrow A$ and of mappings $f_{b}: X_{f(b)} \rightarrow Y_{b}, b \in B$, such that the following condition holds :

$$
\begin{aligned}
(\mathrm{AM})^{*} & (\forall b \in B)\left(\forall C \mathcal{V} \in \mathcal{C}_{o v}\left(C V_{b}\right)\right) \quad\left(\exists b_{0}>b\right) \quad\left(\forall b^{\prime}>b\right) \quad\left(\exists a>f\left(b_{0}\right), f\left(b^{\prime}\right)\right) \\
& \left(\forall a^{\prime}>a\right) \quad\left(q_{b b_{0}} f_{b_{0}} p_{f\left(b_{0}\right), a^{\prime}}, q_{b b^{\prime}} f_{b^{\prime}} p_{f\left(b^{\prime}\right), a^{\prime}}\right)<\mathcal{V} .
\end{aligned}
$$

The set of all approximate mappings from $\boldsymbol{X}$ to $\boldsymbol{Y}$ is denoted by $\operatorname{AP}(\boldsymbol{X}, \boldsymbol{Y})$.

One can easily see that $\operatorname{AP}(\mathscr{X}, \mathscr{g}) \subseteq \operatorname{AP}(\boldsymbol{X}, \boldsymbol{Y})$, whenever $\boldsymbol{X}$ and $\boldsymbol{Y}$ are obtained by omitting the meshes of $\mathscr{X}$ and of respectively. 
Secondly, as a natural generalization of (1.8) onto the nongauged case, we define

(4.2) $\boldsymbol{f} \sim \boldsymbol{f}^{\prime}=\left\{f^{\prime}, f_{b}^{\prime} \mid b \in B\right\}: \boldsymbol{X} \rightarrow \boldsymbol{Y}$, provided the following condition is satisfied :

$$
\begin{array}{cccccc}
(\mathrm{EM}) * & (\forall b \in B) \quad\left(\forall \mathcal{V} \in \mathcal{C}_{o v}\left(Y_{b}\right)\right) \quad\left(\exists b_{0}>b\right) & \left(\forall b^{\prime}>b\right) \quad\left(\exists a>f\left(b^{\prime}\right), f^{\prime}\left(b^{\prime}\right)\right) \\
& \left(\forall a^{\prime}>a\right) & \left(f_{b^{\prime}} p_{f\left(b^{\prime}\right), a^{\prime}}, f_{b^{\prime}}^{\prime} p_{f^{\prime}\left(b^{\prime}\right), a^{\prime}}\right)<q_{b b^{\prime}}^{-1}(\mathcal{V}) .
\end{array}
$$

The relation $\sim$ is indeed an equivalence relation on the set $\operatorname{AP}(\boldsymbol{X}, \boldsymbol{Y})$. The quotient set $\operatorname{AP}(\boldsymbol{X}, \boldsymbol{Y}) / \sim$ is denoted by $\operatorname{AP}[\boldsymbol{X}, \boldsymbol{Y}]$, and the equivalence class of $\boldsymbol{f}$ by $[\boldsymbol{f}]$.

Again an easy testing shows that, if $X$ and $Y$ are $\mathscr{X}$ and $\mathscr{y}$ without the meshes respectively, then $\boldsymbol{f} \sim \boldsymbol{f}^{\prime}: \mathscr{X} \rightarrow \mathscr{g}$ implies $\boldsymbol{f} \sim \boldsymbol{f}^{\prime}: \boldsymbol{X} \rightarrow \boldsymbol{Y}$. Moreover, if $B$ is cofinite, then the converse also holds.

(4.3) Let $\boldsymbol{f}=\left\{f, f_{b} \mid b \in B\right\}: \boldsymbol{X} \rightarrow \boldsymbol{Y}$ be an approximate mapping. Then the formal composition

$$
\mathscr{X}^{*} \stackrel{1}{\longrightarrow} \boldsymbol{X} \stackrel{f}{\longrightarrow} \boldsymbol{Y} \stackrel{1_{*}}{\longrightarrow} y^{*}=\left(Y_{\mu}^{*}, \mathcal{V}_{\mu}^{*}, q_{\mu \mu^{\prime}}^{*}, M^{*}\right),
$$

where $1=\left\{\sigma, 1_{X_{a}} \mid a \in A\right\}, 1_{*}=\left\{t, 1_{Y_{t(\mu)}^{*}} \mid \mu \in M^{*}\right\}, \sigma: A \rightarrow \Lambda^{*}$ is a section of $s: \Lambda^{*} \rightarrow A$ and $t: M^{*} \rightarrow B$ comes from the construction of $a f^{*}$, defines the collec-

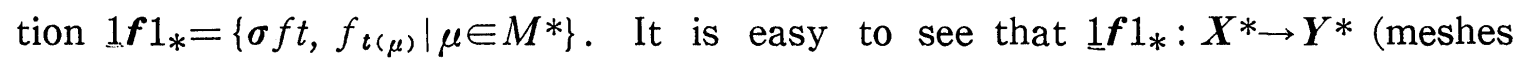
forgotten) is an approximate mapping. Using appropriate terminal shifts (see [7], (7.4) and (7.5)), one can obtain a gauged approximate mapping $v_{2} v_{1}\left(1 \mathrm{f} 1^{*}\right)$ $=f^{*}: \mathfrak{X}^{*} \rightarrow \mathfrak{y}^{*}$. Here, $v_{1}: M^{*} \rightarrow M^{*}$ is defined by means of (A2) and (A3) of $\mathscr{y}^{*}$, which $v_{2}: M^{*} \rightarrow M^{*}$ is defined by means of (AM)* of $\boldsymbol{f}$.

This construction is natural and has functorial properties, i.e., $\boldsymbol{f} \sim \boldsymbol{f}^{\prime}$ if and only if $\boldsymbol{f} * \sim \boldsymbol{f}^{\prime *}$, and $\underline{1}^{*} \sim \underline{1}_{\mathfrak{x}} *$. The proof of these facts is rather long, but not complicated, and we omit it.

Furthermore, if $\boldsymbol{f}: \mathcal{X} \rightarrow \mathscr{Y}$ and $\boldsymbol{g}: \mathscr{Y} \rightarrow \mathcal{Z}$ are interpreted as $\boldsymbol{f}: \boldsymbol{X} \rightarrow \boldsymbol{Y}$ and $\boldsymbol{g}: \boldsymbol{Y} \rightarrow \boldsymbol{Z}$ respectively (meshes forgotten), then

$$
\left[(\boldsymbol{g} \boldsymbol{f})^{*}\right]=\left[\boldsymbol{g}^{*}\right][\boldsymbol{f} *],
$$

whenever $\boldsymbol{g} \boldsymbol{f}: \mathfrak{X} \rightarrow \mathscr{Z}$ exists.

(4.4) The facts from the above allow to define the category APRES, with the objects all cofinite approximate resolutions consisting of topologically complete spaces, and with the sets of morphisms $\underline{\operatorname{APRES}}(\boldsymbol{X}, \boldsymbol{Y})=\operatorname{AP}\left[\boldsymbol{X}^{*}, \boldsymbol{Y}^{*}\right]$ (meshes forgotten). Moreover, if APRES is the category from [7], Theorem 
(8.12), then one can show that the meshes forgetting functor

$$
F: \text { APRES } \longrightarrow \underline{\text { APRES }}, \quad F(\mathscr{X})=\boldsymbol{X} \text { and } F([\boldsymbol{f}])=[\boldsymbol{f}],
$$

is an equivalence of categories.

\section{References}

[1] M.G. Charalambous, Approximate inverse systems of uniform spaces and an application of inverse systems, Comment. Math. Univ. Carolinae 32 (1991), 551-565.

[2] R. Engelking, General Topology, Monografie Matematiczne 60, Polish Scientific Publishers, Warszawa, 1977.

[3] S. Mardešić, Inverse limits and resolutions, in Shape Theory and Geometric Topology, Proc. (Dubrovnik, 1981), Lecture Notes in Math., No. 870, Springer Verlag, Berlin 1981, 239-252.

[4] S. Mardešić, On approximate inverse systems and resolutions, Fund. Math. 142 (1993), 241-255.

[5] S, Mardešić and L. R. Rubin, Approximate inverse systems of compacta and covering dimension, Pacific J· Math. 138 (1989), 129-144.

[6] S. Mardešić and N. Uglešić, Approximate inverse systems which admit meshes, Topology Appl. 59 (1994), 179-188.

[7] S. Mardešić and T. Watanabe, Approximate resolutions of spaces and mappings, Glasnik Mat. 24(44) (1989), 587-637.

[8] N. Uglešic, Isomorphisms in the category of approximate systems, Glasnik Mat. 28(48) (1993), 81-97.

[9] T. Watanabe, Approximative Shape I, Tsukuba J. Math. 11 (1987), 17-59.

Department of Mathematics

University of Split

Teslina $12 / \mathrm{III}$

58,000 Split, Croatia 